Pacific Journal of Mathematics

THE MAUTNER PHENOMENON FOR GENERAL UNITAR 


\title{
THE MAUTNER PHENOMENON FOR GENERAL UNITARY REPRESENTATIONS
}

\author{
Calvin C. Moore \\ Dedicated to Gerhard Hochschild on the occasion of his 65th birthday
}

The 'Mautner phenomenon' for unitary representations is a general assertion of the form that if $x(t)$ is a one parameter subgroup of a Lie group $G, \pi$ a unitary representation of $G$ on a Hilbert space $\mathscr{H}=\mathscr{\mathscr { C }}(\pi)$ and $v$ a vector in $\mathscr{C}$ which is fixed by $x(t)$; i.e., $\pi(x(t)) v=v$, then $v$ must also be fixed by a generally much larger subgroup $H$ of $G$. How much larger $H$ is than the original one parameter group depends in a general way on how noncommutative the group $G$ is. Our purpose here is to establish a very general result of this nature which we believe to be the best possible result of this kind.

1. The 'Mautner phenomenon' for unitary representations is a general assertion of the form that if $x(t)$ is a one parameter subgroup of a lie group $G, \pi$ a unitary representation of $G$ on a Hilbert space $\mathscr{K}=\mathscr{K}(\pi)$ and $v$ a vector in $\mathscr{H}$ which is fixed by $x(t)$; i.e., $\pi(x(t)) v=v$, there $v$ must also be fixed by a generally much larger subgroup $H$ of $G$. How much larger $H$ is than the original one parameter group depends in a general way on how noncommutative the group $G$ is. Our purpose here is to establish a very general result of this nature which we believe to be the best possible result of this kind. The first appearance of such results was in [12] clarifying earlier work in [7], and this theme has appeared in a number of subsequent papers, notably [1], [2], [13], [14], [3], [8], [6], and [15]. The initial application of such results was to the ergodic theory of homogeneous flows, and as can be seen from the other works cited, these applications to ergodic theory have remained central. More recently such results have found applications in the general area of automorphic forms on reductive groups, and more generally the results embodied in the general Mautner phenomenon reflect an interesting and it seems significant property of unitary representations of general Lie groups. In a subsequent papar [5] it will be shown how to use the results here to obtain very comprehensive results on the ergodic theory of homogeneous flows.

Let us now formulate the main result. If $G$ is a connected Lie group, $\mathfrak{g}$ its Lie algebra and $X \in \mathfrak{g}$, we shall say that $X$ (or the corresponding one parameter subgroup $x(t)=\exp (t(X))$ is ad-compact if $\mathrm{Ad}(x(t))$ acting linearly on $\mathfrak{g}$ is contained in a compact subgroup of the full automorphism group of $\mathfrak{g}$. This is clearly equivalent to 
the condition that ad $(X)$ should be semi-simple as linear transformation of $\mathfrak{g}$ with all eigenvalues purely imaginary. If $x(t)$ is given and if $H_{1}$ and $H_{2}$ are two connected normal subgroups of $G$ such that the image of $x(t)$ in $G / H_{i}(i=1,2)$ is ad-compact for $i=1,2$ then the image of $x(t)$ in $G /\left(H_{1} \cap H_{2}\right)$ is easily seen to be ad-compact. Consequently there is a unique smallest connected normal subgroup $H_{x}$ of $G$ such that the image of $x(t)$ in $G / H_{x}$ is ad-compact in $G / H_{x}$. We denote by $\mathfrak{h}_{x}$ the corresponding ideal in $\mathfrak{g}$ and call $H_{x}\left(\mathfrak{h}_{x}\right)$ the ad-compact subgroup (ideal) of $X$. We shall also drop the subscript $x$ when it can be inferred from the context. With this terminology the result is as follows.

TheOREM 1.1. Let $X \in \mathfrak{g}$, the Lie algebra of $G$ and let $H_{x}$ be its ad-compact subgroup, and let $\pi$ be a unitary representation of $G$ on $\mathscr{Y}=\mathscr{H}(\pi)$. Let $v \in \mathscr{H}$ be an eigenfunction for $\pi(x(t))$ i.e., $\pi(x(t)) v=$ $\exp (i \lambda t) v$ for some real $\lambda$. Then $\pi(h) v=v$ for all $h \in H_{x}$. Indeed more is true; if $d \pi(X)$ is the infinitesimal generator of $\pi(x(t))$, then the restriction of $d \pi(X)$ to the orthogonal complement $\mathscr{H}\left(H_{X}\right)$ of the $\pi\left(H_{x}\right)$ fixed vectors in $\mathscr{H}$ has absolutely continuous spectrum.

We recall that a (n unbounded) self adjoint operator, such as $d \pi(X)$ restricted to some invariant subspace, has absolutely continuous spectrum if the corresponding projection valued measure $P(\cdot)$ defined on the Borel sets of the real line satisfies $P(E)=0$ for any Lebesgue null set $E$. It might happen in the theorem that all vectors in $\mathscr{Y}(\pi)$ are fixed by $H_{X}$ so that $d \pi(X)$ on the orthogonal complement of the $H_{X}$ fixed vectors is the zero operator which has absolutely continuous spectrum. Occasionally below we shall talk about an operator having Lebesgue spectrum; this means that the projection valued measure $P$ associated to the operator has the property that $P(E)=0$ iff $E$ is a Lebesgue null set. It will also simplify matters terminologically to agree also that the zero operator on a zero dimensional space has Lebesgue spectrum.

With regard to the two statements of the theorem, let $\mathscr{H}(\pi)=$ $V \oplus \mathscr{H}\left(H_{X}\right)$ be the decomposition of $\mathscr{H}(\pi)$ into the $H_{X}$ invariant vectors and its orthogonal complement $\mathscr{C}\left(H_{X}\right)$. Then as $H_{X}$ is normal, both spaces are $G$-invariant and in particular are $d \pi(X)$ invariant. Let $P$ be the projection valued measure associated with $d \pi(X)$ restricted to $\mathscr{H}\left(H_{X}\right)$. The first statement of the theorem says simply that $P$ has no point masses (i.e., eigenvectors) while the second says in addition that $P$ has no singular continuous part either. Thus if one takes the view that singular continuous spectrum is very unlikely to occur at all in this context, the second statement of the theorem is in some sense almost the same as the first. 
The theorem puts very strict control on the spectrum of $d \pi(X)$ in $\mathscr{H}\left(H_{X}\right)$. However a moment's thought will convince one that there is really no hope of controlling the spectrum of $d \pi(X)$ in the remainder, $V$. In fact our theorem is best possible in that we will show that there is a family of irreducible representation $\lambda$ of $G / H_{X}$ which separates points, such that $d \lambda(X)$ has pure point spectrum for every $\lambda$. Moreover if $\operatorname{Ad}\left(G / H_{X}\right)$ is closed, then one shows easily that any irreducible representation of $G / H_{X}$ has this property.

We note that for semi-simple groups the main theorem above was established in [13] with slightly different terminology. Also our result is closely related to the Theorem 6.1 of [8] and indeed one may deduce the first statement of our theorem in case $G$ is a real algebraic group from the results in [8]. In order to do this one needs a small complement to the results in [8] which may be useful in other contexts. This is given in the final section ( $\$ 6$ ). The strategy in the proof of Theorem 1.1 is inspired by and in parts follows closely the work of Dani [6]. It is pleasure to acknowledge our debt to Dani's work, and in particular to his idea as to how to break apart the problem into a semi-simple one and a solvable one. The remainder of the paper with the exception of the final section $(\S 6)$ is devoted to the proof of Theorem 1.1. The proof proceeds by a series of reductions; some of intermediate results are isolated as separate theorems as they have independent interest.

We would also like to thank Jon Brezin who contributed much to our thinking by many discussions and the work in [5].

2. There are several easy observations that will be used throughout. First we may always assume that $G$ is simply connected for we just replace any given $G$ by its universal covering group and then lift the representation $\pi$ back. One has to take care to note that the ad-compact subgroup $\widetilde{H}_{X}$ associated to $X$ in $\widetilde{G}$ may not project onto the corresponding subgroup $H_{X}$ of $G$, but in any case it is clear that the image of $\widetilde{H}_{X}$ is dense in $H_{X}$ and so the $H_{X}$ fixed vectors are the same as the $\widetilde{H}_{X}$ fixed vectors. Henceforth therefore we will always assume that $G$ is simply connected; recall then that every normal analytic subgroup is closed.

Secondly we observe that if we know that Theorem 1.1 holds for every irreducible representation of a given group $G$, it holds for all representations. One simply decomposes a general representation as a direct integral of irreducible representations, and it is an easy exercise to check that the validity of theorem for each integrand implies the validity for the integral.

The third observation, contained in the lemma below, allows us to factor out connected normal subgroups in the kernel of a given 
representation. We omit the simple proof.

LEMMA 2.1. Let $p$ be a surjection of the simply connected $G$ onto a simply connected $G^{\prime}$. Let $X$ be in the Lie algebra g of $G$ and $X^{\prime}=d p(X)$ its image in $\mathfrak{g}^{\prime}$; let $H_{X^{\prime}}$ and $H_{X^{\prime}}$ be the corresponding ad-compact subgroups of $G$ and $G^{\prime}$. Then $H_{X^{\prime}}=p\left(H_{X}\right)$.

The fourth and final initial observation is contained in the following lemma and will be used repeatedly. We again omit the proof.

LEMMA 2.2. If $A$ is a self adjoint operator on a Hilbert space $\mathscr{K}$ and if $\mathscr{K}_{\alpha}$ is family of invariant subspaces such that $A \mid K_{\alpha}$ has absolutely continuous spectrum for each $\alpha$, then $A \mid \mathcal{C}$ has absolutely continuous spectrum where $\mathscr{H}$ is the closed linear span of $Y_{a}$. If in addition the spectrum is Lebesgue on at least one "Y, it is Lebesgue on $\mathscr{E}$.

We now come to the proof of Theorem 1.1; of course we prove the second statement as the first is a corollary of it. The first step in the reduction goes back to [12] and the argument for the first statement of this step of the proof of the theorem is well known; the stronger version we need appears almost as we want it in [15] (we thank A. Katok for calling this paper to our attension) and something very much like it appears in [6]. For completeness we include the straightforward argument. We shall systemically use the following notation; for a unitary representation $\pi$ of $G$ on a Hilbert space $\mathscr{C}=\mathscr{K}(\pi)$, and a closed subgroup $K$ of $G, \mathscr{K}(K)$ will be the orthogonal complement of the $K$-fixed vectors.

We consider the generalized eigenspaces $\mathfrak{g}^{\alpha}(\alpha \in C)$ of ad $(X)$ acting on $g$ where $\mathfrak{g}^{\alpha}=\left\{Y:(\operatorname{ad}(X)-\alpha)^{n} Y=0\right.$ for some $\left.n\right\}$. Since $\left[\mathfrak{g}^{\alpha}, \mathfrak{g}^{\beta}\right] \subset \mathfrak{g}^{\alpha+\beta}$ it follows that the subalgebra $\mathfrak{p}=\mathfrak{p}_{X}$ generated by the $\mathfrak{g}^{\alpha}$ with $\operatorname{Re}(\alpha) \neq 0$ is in fact an ideal. Let $P$ be the corresponding normal subgroup. Note that $\mathfrak{p}$ is the smallest ideal of $g$ such that ad $(X)$ has purely imaginary eigenvalues on $\mathfrak{g} / \mathfrak{p}$. The first reduction is the following.

THEOREM 2.3. If $\pi$ is a unitary representation of $G$, then the spectrum of $d \pi(X)$ in the orthogonal complement $\mathscr{Y}(P)$ of the $P$ fixed vectors is Lebesgue.

Proof. We let $\mathfrak{p}^{ \pm}$be the subalgebras generated by the $\mathrm{g}^{\alpha}$ with $\operatorname{Re}(\alpha)>0 \quad(<0)$ and $P^{ \pm}$the corresponding subgroups. It is clear that $P^{ \pm}$are simply connected nilpotent groups, and that $P$ is generated by $P^{+}$and $P^{-}$. If as above $\mathscr{C}\left(P^{ \pm}\right)$and $\mathscr{C}(P)$ are the orthogonal complements of the $P^{ \pm}$and $P$ fixed vectors respectively, 
then it is clear that $\mathscr{H}(P)$ is the closed linear span of $\mathscr{H}\left(P^{+}\right)$and $\mathscr{H}\left(P^{-}\right)$. Consequently by Lemma 2.2 it suffices to show that $d \pi(X)$ has Lebesgue spectrum in $\mathscr{C}\left(P^{ \pm}\right)$, and by symmetry it suffices to consider $\mathscr{H}\left(P^{+}\right)$. Actually we shall show that for any connected ad $(X)$ invariant subgroup $Q$ of $P^{+}$, the spectrum of $d \pi(X)$ in $\mathscr{H}(Q)$ is Lebesgue. The argument is by induction on the dimension of $Q$, the case $Q=(e)$ being clear.

We choose some $Q$ and assume that the theorem is known for all $Q^{\prime}$ of smaller dimension. Now it is clear that we can find an ad $(X)$ invariant connected subgroup $Q^{\prime}$ of $Q$ such that the action of $\operatorname{ad}(X)$ on the quotient of the Lie algebras $\mathfrak{q} / \mathfrak{q}^{\prime}$ is irreducible with an eigenvalue that is not pure imaginary. Since $d \pi(X)$ has by induction Lebesgue spectrum on $\mathscr{H}\left(Q^{\prime}\right)$, it follows from Lemma 2.2 that $d \pi(X)$ will have Lebesgue spectrum on $\mathscr{H}(Q)$ provided that it has Lebesgue spectrum on $V=\mathscr{H}(Q) \ominus \mathscr{C}\left(Q^{\prime}\right)$. Let $S$ be the semi direct product of $Q$ and the one parameter group $x(t)$. Then $\pi(S)$ preserves $V$ and $\pi\left(Q^{\prime}\right)$ acts trivially and so $\pi$ defines a representation $\pi_{1}$ of the quotient $S / Q^{\prime}$. Moreover this representation has no $Q / Q^{\prime}$ fixed vectors. The group $S / Q^{\prime}$ is solvable and is either the ' $a x+b$ ' group or is a three dimensional solvable group with one root which is not pure imaginary. In either case by the Mackey theory any irreducible representation $\lambda$ with no $Q / Q^{\prime}$ fixed vectors is easily seen to be induced by a character of $Q / Q^{\prime}$. It follows that $d \lambda(X)$ has Lebesgue spectrum (with uniform multiplicity one). Since our representation $\pi_{1}$ is a direct integral of such irreducible $\lambda$, it follows that $d \pi_{1}(X)$, and hence $d \pi(X)$ has Lebesgue spectrum in $V$. This completes the proof of Theorem 2.3.

It follows from Theorem 2.3 and Lemma 2.2 that it suffices to consider only representations $\pi$ of $G$ in which the subgroup $P$ acts trivially. By Lemma 2.1 we may factor out $P$, or in other words we are free to, and will assume that all eigenvalues of $\operatorname{ad}(X)$ are pure imaginary.

3. The next reduction in the proof of Theorem 1.1 will be to the even more special case when ad $(X)$ has only zero as eigenvalue that is, $\operatorname{ad}(X)$ is nilpotent. This section is devoted to that reduction.

We consider Ad $(x(t))$ as a subgroup of the automorphisms group of the Lie algebra $g$ of $G$. Let $A$ denote the algebraic hull of this one parameter group; then $A$ is a connected abelian algebraic linear group and consequently $A$ can be written as a product $A=U \times C$ where $U$ is unipotent and $C$ is an algebraic torus [4]. Since $\operatorname{Ad}(x(t))$ has eigenvalues on the unit circle, it follows that $C$ is compact. Then as $G$ is simply connected, any automorphism of $\mathfrak{g}$ 
defines an automorphism of $G$ and so $C$ may be regarded as a group of automorphisms of $G$ and we could form the semi direct product $G \cdot C$. However since we have agreed to consider only simply connected groups, let $\widetilde{C}$ be the universal covering group of $C$; then we form the semi direct $G^{\prime}=G \cdot \widetilde{C}$ (which is the universal covering group of $G \cdot C$ ).

Let $g^{\prime}=g+c$ be the Lie algebra of $\widetilde{G}$ where $c$ is the Lie algebra of $\widetilde{C}$. In view of the definition of $C$, it is clear that a subalgebra of $\mathfrak{g}$ is an ideal in $\mathfrak{g}$ if and only if it is an ideal in the larger algebra $\mathfrak{g}^{\prime}$. Consequently if $\mathfrak{h}_{X}$ is the ad-compact ideal of $X$ viewed as an element of $\mathfrak{g}$, then $\mathfrak{h}_{X}$ is an ideal in $\mathfrak{g}^{\prime}$. Moreover as c commutes with $X$ by its construction, and $\mathfrak{g}^{\prime}=\mathfrak{g}+\mathfrak{c}$, it follows that the image of $X$ in $\mathfrak{g}^{\prime} / \mathfrak{h}_{X}$ is ad-compact. Now if $\mathfrak{h}_{X}^{\prime}$ is the adcompact ideal of $X$, viewed as an element of $\mathfrak{g}^{\prime}$, it is clear on general principles that $\mathfrak{h}_{X}^{\prime} \supset \mathfrak{h}_{X}$. But the above establishes the reverse inclusion, and so $\mathfrak{h}_{X}^{\prime}=\mathfrak{h}_{X}$. Thus dropping the subscript $X$, we see that the ad-compact subgroup $H^{\prime}$ of $x(t)$ in $G^{\prime}$ sits inside $G$ and coincides with the adcompact subgroup of $x(t)$ computed relative to $G$.

Now if $\pi$ is any representation of $G$ on a Hilbert space $\mathscr{K}$ let $\pi^{\prime}=U^{\pi}$ be the representation of $G^{\prime}$ induced by $\pi$. Let $\mathscr{H}^{\prime}$ be its Hilbert space. Then the restriction of $\pi^{\prime}$ to $G$ is either by direct computation in this simple case, or Mackey's subgroup theorem [10], a direct integral of conjugates $\pi^{c}$ of $\pi$ by elements $c$ of $C$. Since $H=H^{\prime} \subset G$ from above, we see that the orthogonal complement $\mathscr{C}^{\prime}\left(H^{\prime}\right)$ of the $\pi^{\prime}\left(H^{\prime}\right)$ fixed vectors in $H^{\prime}$ is also simply the direct integral of the spaces $\mathscr{H}^{c}(H)$ of the orthogonal complements of the $\pi^{c}(H)$ fixed vectors in the Hilbert space $\mathscr{H}^{c}$ of $\pi^{c}$. But $\mathscr{H}^{c}$ can be identified with $\mathscr{H}\left(\left(\right.\right.$ as $\left.\left.\pi^{c}(g)=\pi\left(c g c^{-1}\right)\right)\right)$ and then $\mathscr{H}^{c}(H)=\mathscr{H}(H)$ as $H$ is normalized by $C$. The upshot is that $\mathscr{H}^{\prime}\left(H^{\prime}\right)$ is simply a direct integral of copies of $\mathscr{H}(H)$.

Moreover as $C$ commutes with $x(t)$ by construction, the infinitesimal generator $d \pi^{c}(X)$ is exactly the same as the infinitesimal generator $d \pi(X)$, and the same holds for their restrictions to $\mathscr{C}(H)$ and $\mathscr{H}^{c}(H)$. Finally we see that $d \pi^{\prime}(X)$ restricted to the subspace $\mathscr{K}^{\prime}\left(H^{\prime}\right)$ is simply the direct integral of copies (indexed by $c \in C$ ) of $d \pi(X)$ restricted to $\mathscr{C}(H)$. That is, $d \pi^{\prime}(X)$ in $\mathscr{C}^{\prime}\left(H^{\prime}\right)$ is unitarily equivalent to a multiple of $d \pi(X)$ in $\mathscr{C}(H)$, and hence their spectral measures have the same type. The end result of this analysis is that the validity of Theorem 1.1 for the group $G$ (for the element $X \in \mathfrak{g} \subset \mathfrak{g}^{\prime}$ ) implies its validity for the group $G^{\prime}$ (for the element $X$ ).

But now since $C$ commutes with $x(t)$, the group $B$ generated by $x(t)$ and $\widetilde{C}$ in $G^{\prime}$ is abelian. Its Lie algebra $\mathfrak{b}$ is the span of $c$ and $X$. By the construction of $C$ as the semi-simple part of the algebraic hull of $\operatorname{Ad}(x(t))$, we see that we can write a decomposition of $X$ in 
$\mathfrak{b}$ as $X=Y+W$ where $W$ lies in the Lie algebra $\mathfrak{c}$ of $\widetilde{C}$ and where ad $(Y)$ operating on $\mathfrak{g}^{\prime}$ is nilpotent; in fact ad $(Y)$ on $\mathfrak{g}$ is simply the nilpotent part of ad $(X)$. Now $W$ is clearly seen by construction to be ad-compact in $G^{\prime}$ and consequently as $X, Y$, and $W$ commute, the image of $X$ in any quotient of $G^{\prime}$ will be ad-compact iff the image of $Y$ in the same quotient is ad-compact. Consequently the ad-compact subgroups $H_{X}^{\prime}$ and $H_{Y}^{\prime}$ in $G^{\prime}$ coincide.

Furthermore as we have already noted, it suffices to prove Theorem 1.1 for irreducible representations of $G^{\prime}$. Consequently to check Theorem 1.1 it suffices to consider irreducible representations $\pi^{\prime}$ of $G^{\prime}$. If $\pi^{\prime}$ is such, then $d \pi^{\prime}(X)=d \pi^{\prime}(Y)+d \pi^{\prime}(W)$; but since $\exp (t W)$ is by construction contained in a subgroup of $G^{\prime}$ which is compact mod the center of $G^{\prime}$, it follows that $d \pi^{\prime}(W)$ has discrete spectrum. Then as in [6] Lemma 1.9, it follows that $d \pi^{\prime}(X)$ has absolutely continuous spectrum iff $d \pi^{\prime}(Y)$ does. The upshot of this is that the validity of Theorem 1.1 for $G^{\prime}$ and the element $X$ of its Lie algebra follows from the validity of Theorem 1.1 for $G^{\prime}$ and the element $Y$ of its Lie algebra (and conversely). But we have already observed that ad $(Y)$ is nilpotent on $\mathfrak{g}^{\prime}$, and consequently we have shown that it suffices to prove Theorem 1.1 when the element $X$ in the Lie algebra of the group in question is nilpotent i.e., $\operatorname{ad}(X)$ is a nilpotent derivation of $\mathfrak{g}$.

4. We now consider a special case of the above, namely when $X$, which is already assumed to be nilpotent, actually lies in $n$ the Lie algebra of the nilradical $N$ of $G$. This special case is important for two reasons; first we will in fact end up the next section reducing the general theorem for ad $(X)$ nilpotent to this case, and secondly this special case will have a corollary that will be used in that reduction. Thus we now prove the following.

THeOREM 4.1. If $x(t)=\exp (t X)$ belongs to the nilradical $N$ of $G$ (i.e., $X \in \mathfrak{n}$ ), then $H_{X} \subset N$ (i.e., $\mathfrak{h}_{X} \subset \mathfrak{n}$ ) and if $\pi$ is any representation on a Hilbert space $\mathscr{H}$, the spectrum of $d \pi(X)$ on $\mathscr{C}\left(H_{X}\right)$, the orthogonal complement of the $H_{X}$ fixed vectors is absolutely continuous.

The corollary that we want is the following fact which is an extension of Lemma 4.2 of [6] and seems of some independent interest.

TheOREM 4.2. For $M$ a connected subgroup of $N$, let $\widetilde{M}$ be the smallest normal subgroup of $G$ containing $M$. Then if $\pi$ is any unitary representation of $G$ and $v$ is vector such that $\pi(m) v=\theta(m) v$ 
for $m \in M$, where $\theta$ is some character of $M$, then $\pi(\widetilde{m}) v=\tilde{\theta}(\tilde{m}) v$ for $\widetilde{m} \in \widetilde{M}$ where $\tilde{\theta}$ is a character of $\widetilde{M}$. If $\theta=1$, then $\tilde{\theta}=1$ also.

We first show how to deduce (4.2) from (4.1). To do this let $x(t)=\exp (t X)$ be any one parameter subgroup of $M$, and let $H_{X}$ be the corresponding ad-compact subgroup of $G$. By (4.1) $\pi\left(H_{X}\right)$ must fix $v$. Thus if we replace $\pi$, as we may, by the cyclic subrepresentation generated by $v$, we see that $H_{X}$ must act trivially on $\mathscr{H}=\mathscr{H}(\pi)$. Thus we can simply divide out all those $H_{X}$ and so assume that $H_{X}=(e)$ for every $X \in \mathfrak{m}$. But then every $X$ in $\mathfrak{m}$ is ad-compact, and since ad $(X)$ is nilpotent, this must mean that $X$ is central. Thus $\mathfrak{m}$ is central and hence $M$ is also, and finally $M=\widetilde{M}$; this completes the derivation of (4.2) from (4.1).

For the proof of (4.1), note that if $X \in \mathfrak{n}$, then ad $(X)$ acts trivially on $\mathfrak{g} / \mathfrak{n}$ and so by the definition of $\mathfrak{h}_{X}, \mathfrak{h}_{X} \subset \mathfrak{n}$. As we have noted already we may assume that the given unitary representation $\pi$ on $\mathscr{H}$ is irreducible. Then as $H_{X}$ is normal, $\mathscr{C}\left(H_{X}\right)$ is $G$-invariant. Therefore to prove (4.1) we have to establish a dichotomy: either $d \pi(X)$ is absolutely continuous (on all of $\mathscr{H}$ ) or $\pi$ annihilates $H_{X}$. We are also free to assume that the kernel of $\pi$ is discrete for we can divide out the connected component of its kernel. We claim in fact now that either $d \pi(X)$ is absolutely continuous or else $X$ is central. This will do the trick for if $X$ is central, $H_{X}=(e)$ and $\pi$ annihilates it automatically.

We shall apply the Mackey and Kirillov theories to $\pi$, [11], [9]. We first restrict the irreducible $\pi$ to the normal type I subgroup $N$, and decompose it as a direct integral of irreducibles. This produces a measure $\nu$ on the unitary dual $\hat{N}$ of $N$ which is ergodic under the natural action of $G$. But now $\hat{N}$ is simply the quotient $n^{*} / \mathrm{ad}^{*}(N)$ by the Kirillov theory, and the measure $\nu$ can be lifted up to measure $\mu$ on $\mathfrak{n}^{*}$ which is ergodic and quasi-invariant under the coadjoint action of $G$ on $\mathfrak{n}^{*}$. (To define $\mu$, one can take $\nu$ to be finite and then choose in a smooth way finite measures $\mu_{0}$ on each orbit of $N$ on $\mathfrak{n}^{*}$ which are quasi-invariant, and then define $\mu$ to be the integral of the fiber measures $\mu_{0}$ with respect to the base measure $\nu$ on the space of orbits.)

Let the one dimensional space generated by $X \in \mathfrak{n}$ be denoted by $\mathfrak{m}$, (if $X=0$, there is nothing to prove) and let $\mathfrak{n t}^{\perp}$ be its annihilator in $\mathfrak{n}^{*}$. The one dimensional quotient $\mathfrak{n}^{*} / \mathfrak{m}^{\perp}$ can be identified canonically with the dual vector space $\mathfrak{m}^{*}$ to $\mathfrak{m}$, and hence with the dual group to the one parameter group $\exp (t X)=x(t)$, which is where the spectral measure $P(\cdot)$ of $d \pi(X)$ lives. Thus $P$ can be viewed as a projection valued measure on $\mathfrak{n}^{*} / \mathfrak{m}^{\perp}$. The basic fact that we need is that if we project the finite measure $\mu$ on $\mathfrak{l t}^{*}$ constructed above 
onto the quotient $\mathfrak{n}^{*} / \mathfrak{n}^{\perp}$ via the projection map $q$, we obtain a scalar measure $q_{*}(\mu)$ on $\mathfrak{n}^{*} / \mathfrak{m}^{\mathrm{L}}$ which is equivalent to the projection valued measure $P$ in the sense that $P(E)=0$ iff $q_{*}(\mu)(E)=0$. If the representation $\pi$ were irreducible on $N$, so that the measure $\mu$ is concentrated on a single orbit, this assertion is essentially in [9] (and in any case a simple induction argument proves it). Once one has the assertion for $\pi$ irreducible on $N$, one can immediately obtain it for any $\pi$ by taking a direct integral decomposition.

Thus what we have to show is that either $q_{*}(\mu)$ is absolutely continuous or else $X$ is central. Now consider some $G$ orbit $G \cdot f$ in $\mathfrak{n}^{*}$ and let $\mu_{f}$ be a finite measure on $G \cdot f$ quasi-invariant under $G$. This orbit is a countable union of small pieces $U_{\imath}$ so that each $U_{i}$ is homeomorphic to an open subset of the homogeneous space $G / G_{f}$ (where $G_{f}$ is the isotropy group of $f$ ) and such that each $U_{i}$ is a connected real analytic submanifold of $n^{*}$. We may also take the measure $\mu_{f}$ to have a real analytic density in $U_{i}$; we let $\left(\mu_{f}\right)_{i}$ be restriction of $\mu_{f}$ to $U_{i}$. Then as the projection $q$ is a real analytic the function on $U_{i}$, it follows by elementary geometry that the projection $q_{*}\left(\mu_{f}\right)_{i}$ of $\left(\mu_{f}\right)_{i}$ is either absolutely continuous or else that $q$ is constant on some open subset of $U_{v}$, and hence constant on $U_{i}$. But now the composition of the map from $G / G_{f}$ onto $G \cdot f$ with $q$ is a real analytic map of the (real analytic) manifold $G / G_{f}$ into the real line. If it is constant on some open set, and as $G$ is connected, it must be constant globally. We therefore conclude that either $q_{*}\left(\mu_{f}\right)_{i}$ is absolutely continuous for all $i$ or else $q$ is constant on the orbit $G \cdot f$. Since a countable sum of absolutely continuous measures is absolutely continuous it follows that $q_{*}\left(\mu_{f}\right)$ is either absolutely continuous or else $q$ is constant on the orbit $G \cdot f$.

But now the set of all $f$ such that $q$ is constant on the $G$-orbit of $f$ is clearly a $G$-invariant set, and by ergodicity of the measure $\because$, either $q$ is constant on $\mu$-almost no orbits or $\mu$-almost all orbits. In the latter case the constant value of $q$ on almost all orbits is a $"$-almost everywhere defined $G$-invariant Borel function. Again by ergodicity, this function must be $\because$-almost everywhere constant. When translated into representation theoretic language, this says that $\pi(x(t)) w=\exp (2 \pi i \lambda t) w$ for all $w$ where $\lambda$ is the constant value of $q$. This implies that the image of $x(t)$ in $G / \operatorname{ker}(\pi)$ is central, but since we have made the assumption that the kernel of $\pi$ is discrete, this easily implies that $x(t)$ is central in $G$, or equivalently $X$ is central in $g$.

What we have shown therefore at this point is that either $q_{*}\left(\mu_{f}\right)$, in the notation above, is absolutely continuous for $"$-almost all $f$ or else $X$ is central. According to our initial comments we are done if we can show that $q_{*}\left(\ell_{f}\right)$ absolutely continuous for $!^{\prime-}$ 
almost $f$ implies that $q_{*}(\mu)$ is absolutely continuous. Recall that $\mu$ is an ergodic $G$ quasi-invariant measure on $\mathfrak{n}^{*}$ and $\mu_{f}$ is (the) quasiinvariant measure on the $G$-orbit of $f \in \mathfrak{n}^{*}$. It is now a simple excercise in ergodic theory to see that indeed $q_{*}(\mu)$ gives zero measure to any set which is a null set for if $q_{*}\left(\mu_{f}\right)$ for $\mu$-almost all $f$. This completes the proof.

5. We resume the study of a general $X \in g$ with ad $(X)$ nilpotent and complete the proof of the theorem. We rely heavily on Dani's argument in $\S \S 4$ and 5 of [6]. We let $\mathfrak{r}$ be the radical of $\mathfrak{g}$ and pick a semi-simple Levi factor $\mathfrak{l}$ so that $\mathfrak{g}=\mathfrak{r}+\mathfrak{l}$ is a semi direct product. Let $\mathfrak{l}=\sum \mathfrak{l}$ be the decomposition of $\mathfrak{l}$ into its simple factors and let $X_{i}$ be the component of $X$ in $\mathfrak{l}_{i}$. Then let $j$ be the sum of all those simple factors $\mathfrak{l}_{i}$ such that $X_{i} \neq 0$. Now following Dani, we define $\mathfrak{m}=\mathfrak{m}(\mathfrak{j})$ to be the largest subalgebra of the nilradical t of $g$ such that $j$ operates trivially on $\mathfrak{r} / \mathfrak{m}$. It is immediate that $\mathfrak{m}$ is in fact an ideal in $\mathfrak{g}$ and is independent of the choice of the Levi factor $\mathfrak{l}$. Let $M=M(\mathfrak{j})$ be the subgroup of $N$ corresponding to $\mathfrak{m}$, and $J$ the subgroup of $G$ corresponding to $i$. The result desired is the following.

THEOREM 5.1. Suppose that $\pi$ is a unitary representation of $G$; then the spectrum of $d \pi(X)$ restricted to $\mathscr{C}(M)$, orthogonal complement of the $M$ fixed vectors is Lebesgue.

Proof. Dani proves precisely this in Proposition 4.1 of [6] but for the representation $\pi$ on $L^{2}(G / C)$ for certain kinds of finite volume homogeneous spaces and for single elements of the group instead of one parameter subgroups. His argument is completely representation theoretic except for a preliminary lemma (Lemma 4.2) needed in the proof, where he uses the geometry of the space $G / C$. However our Theorem 4.2 above gives a proof in complete generality of this essential lemma. With this observation, Dani's argument carries over word for word except that one has to transform the argument a single element into an argument for one parameter group. This for is easy to do, and we do not feel that carrying out the details would give additional insight.

In view of Theorem 5.1 and Lemma 2.2, we can simply assume that $M=M(\mathfrak{j})=(e)$. Then the subgroup $J$ above becomes normal in G. Then Dani's argument in Proposition 5.1 of [6] yields the following.

THEOREM 5.2. In the situation above, the spectrum of $d \pi(X)$ on $\mathscr{C}(J)$, the orthogonal complement of the $J$ invariant vectors, is 
absolutely continuous.

Proof. Again this is a matter of modifying Dani's argument in Proposition 5.1 to apply to one parameter groups instead of single elements. In fact the theorem follows very easily from the main results in [13]. For one considers the reductive group $J^{\prime}$ generated by $J$ and $(x(t))$. Any irreducible representation of $J^{\prime}$ is scalar on the center of $J^{\prime}$ and so is essentially determined by its restriction to $J$ and the results in [13] give the desired result.

Now in view of Theorem 5.2 and Lemma 2.2 again, we may restrict to representations where $J$ acts trivially. Then we can factor $J$ out altogether, and assume $J=0$. But then by the definition of $J$, this means that the projection of our element $X \in \mathfrak{g}$ into $\mathfrak{g} / \mathfrak{x}$, where $\mathfrak{x}$ is the radical, is trivial. Thus $X \in \mathfrak{r}$ but we are also assuming that $\operatorname{ad}(X)$ is nilpotent. This implies then that $X \in \mathfrak{n}$, the nilradical of $g$. But we have already established Theorem 1.1 in this case (Theorem 3.1) and so the proof of Theorem 1.1 is complete.

We mentioned in the introduction that Theorem 1.1 is in a sense the best possible result in this direction. To be more precise, note that statements about the spectrum of $d \pi(X)$ on $\mathscr{C}(H)$, the orthogonal complement of the $\pi(H)$ fixed vectors are stronger statements the larger the subgroup $H$ is. We claim that in Theorem 1.1 is best possible that the subgroup $H_{X}$ is the largest possible subgroup that will work. This follows from the fact that the quotient group $G / H_{X}$ has a separating family of irreducible representations $\pi$ such that $d \pi(X)$ has pure point spectrum. To see this, let $K=G / H_{X}$, and first assume that the adjoint group of $K$ is closed. Then the image of $x(t)$ in the adjoint group of $K$ lies in a compact subgroup of $\operatorname{Ad}(K)$. Consequently the image of $x(t)$ in $K$ lies in a subgroup that is a compact extension of the center of $K$. It follows then that for any irreducible representation $\pi$ of $K, d \pi(X)$ has pure point spectrum.

In general if $\mathrm{Ad}(K)$ is not closed we can play the same game we did in $\S 3$. Namely, let $C$ be the algebraic hull of $\operatorname{Ad}(x(t))$ in the group of all automorphisms of the Lie algebra of $K$. Then by definition $C$ is compact and moreover can be viewed as a group of automorphisms of $K$. We form semi direct product $K^{\prime}=K \cdot C$ and observe that the one parameter group $x(t)$ in $K$ is contained in a subgroup of $K^{\prime}$ which is a compact extension of the center of $K^{\prime}$. Consequently for any irreducible representation $\pi^{\prime}$ of $K^{\prime}, d \pi^{\prime}(K)$ has pure point spectrum. It is an easy matter to see that the restriction of $\pi^{\prime}$ to $K$ remains irreducible and these restrictions provide the 
separating family whose existence was asserted.

6. We noted in the introduction that the first part of Theorem 1.1 could be easily deduced from Theorem 6.1 of [8] in the case when the group $G$ is the topological component of the identity of (the set of real points of) an algebraic group. We show how to do this in this section and as a part of this we give a complement to the results in [8] which is needed here and should be useful in applying the results of [8] in other contexts as well. To state this result recall that the projective kernel of a representation of a group $G$ is the set of all $g \in G$ such that $\pi(g)$ is a scalar multiple of the identity. The projective kernel of an irreducible representation plays a central role in [8], and we need some information about it in the context of [8]. The main fact is that $P$ is very close to being an algebraic subgroup of $G$.

THeORem 6.1. Let $G$ be (the set of real points of) a connected algebraic group and let $\pi$ be an irreducible unitary representation of $G$, with projective kernel $P$. Then $P$ is of finite index in its algebraic hull $\bar{P}$. Moreover if the kernel of $\pi$ contains no connected unipotent algebraic subgroups of $G$, and $U$ is the unipotent radical of $G$, then $P \cap U$ is a vector group central in $G$ and of dimension at most one; moreover $P$ is contained in the centralizer $Z(U)$ of $U$.

We defer the proof of this for a moment and show how to prove the first part of Theorem 1.1 for the topological component of the identity $G_{0}$ of a connected algebraic group $G$. It is easy to see that it suffices to prove (1.1) for $G$ instead of $G_{0}$. We have already seen that it suffices to consider irreducible representations $\pi$ of $G$ in (1.1). Let $P$ be the projective kernel of $\pi$ and let $H_{X}$ be the ad-compact subgroup of $G_{0}$ associated to $X$. (Since $G$ is algebraically connected, it is clear that $H_{X}$ is also normal in G.) Then if $v$ were an eigenvector for $\pi(x(t))$, that is $\pi(x(t)) v=\exp (i \lambda t) v$, then the matrix coefficient $(\pi(x(t)) v, v)$ has constant absolute value. We have to show that $\pi$ annihilates $H_{X}$. But by Theorem 6.1 of [8] the absolute value of this matrix coefficient tends to zero on $G / P$; hence $x(t)$ must remain in a compact set modulo $P$. But by (6.1) the component of the identity $P_{0}$ is of finite index in $P$ and so $x(t)$ is in a compact set modulo $P_{0}$. Now let $K_{0}$ be the component of the kernel of $\pi$. Then $K_{0} \subset P_{0}$ and is of codimension less than or equal to one in $P_{0}$. As we have argued before $P_{0} / K_{0}$ must be central in $G_{0} / K_{0}$ and it follows therefore that the image of the one parameter group $x(t)$ in $G / K_{0}$ is ad-compact. Hence $H=H_{X} \subset K_{0}$ and so $\pi$ annihilates $H_{X}$ as desired. 
We now turn to the proof of Theorem 6.1. Let $K$ be the largest connected normal unipotent algebraic subgroup contained in $\operatorname{ker}(\pi)$. It is not hard to see that the first statement of the theorem for $G$ follows if we can prove it for $G / K$. Therefore we may assume that $K=(e)$. First of all we note that if $G=U$ is unipotent, then a close look at the Kirillov theorem will show immediately that $P$ is connected and hence algebraic. For general $G$, we restrict the given irreducible unitary representation $\pi$ of $G$ to the unipotent radical $U$. Since $U$ is type $I, \pi$ decomposes as direct integral of multiples of irreducible representation $\lambda^{x}$ of $U$ with respect to a measure on $\hat{U}$. Since $G$ is algebraic, it acts smoothly on $\hat{U}$ and so this measure is concentrated on a single orbit of G.

The projective kernel $P^{x}$ of $\lambda^{x}$ is connected by the above and $P \cap U$ must be contained in $U_{1}=\bigcap_{x} P^{x}$. As the intersection of connected subgroups in $U$ is connected, $U_{1}$ is connected. Now clearly $\pi$ maps $U_{1}$ into a commuting family of operators and so $U_{1} /\left(U_{1} \cap \operatorname{ker}(\pi)\right)$ is commutative. On the other hand, $\operatorname{ker}(\pi) \cap U_{1}$ must be discrete for otherwise $\operatorname{ker}(\pi)$ would contain a connected unipotent algebraic subgroup of $G$. Consequently $U_{1}$ is an abelian unipotent group, hence a vector group. Each representation $\lambda^{x}$ is a multiple of a character $\sigma^{x}$ of $U_{1}$. Since $U_{1}$ is normal in $G$, these characters $\sigma^{x}$ constitute a single orbit $O$ of the action of $G$ on $\hat{U}_{1}$ which we identify with the dual vector space. It is clear that $P \cap U$ is precisely that subgroup of $U_{1}$ consisting of those $u$ such that $\sigma(u)$ is constant as $\sigma$ runs over the orbit $\odot$. Now just as in [8], the fact that $G$ is connected as algebraic group implies that $P \cap U$ is connected, and hence a subvector group of $U_{1}$. It is immediate moreover that $P \cap U$ has dimension at most one. If it is trivial, it is central in $G$ and if not trivial, $\operatorname{ker}(\pi)$ meets it in a discrete subgroup $D$ and $(P \cap U) / D$ is central in $G / D$. It then follows that $P \cap U$ is central in $G$ as desired.

We now look at $P \cap R$ where $R$ is the radical of $G$; since $P \cap U$ is centralized by $G$ and hence $R$, it follows that $P \cap R$ is nilpotent. Then its algebraic hull $\overline{P \cap R}$ is also nilpotent, and of course normal in $R$. Then the algebraic component of the identity of $\overline{P \cap R}$ is normal in $R$ and may be written as a direct prodeut $V \times T$ where $V$ is its unipotent radical and $T$ is the unique maximal torus. But if $V \times T$ is to be normal in $R, T$ must necessarily act trivially on $U / V$. Since $T$ already centralizes $V$, it follows by complete reducibility that $T$ centralizes $U$. Since $T$ also centralizes a Levi factor for $U$ in $G$, it follows that $T$ is central in $G$. But then $T$ is already inside the projective kernel $P$ of our representation. It follows immediately that $V$ is the algebraic hull of $P \cap U$; in other words $V=P \cap U$ 
and is central in $G$. It follows that $P \cap R=\widetilde{P \cap R}$ is algebraic and that its algebraic compotent of the identity has the form $(P \cap U) \times T$ where $T$ is an algebraic torus central in $G$ : in fact $T$ is necessarily the largest central torus in $G$.

Finally we turn to the part of $P$ outside $R$. Observe that $R \cdot P / R$ is an analytic normal subgroup of $G / R$. Therefore up to finite groups ( $G / R$ may be topologically disconnected) it is a sum of some of the simple factors of $G / R$. It follows now that the component of the identity $P_{0}$ of $P$ is of finite index on $P$ and is an extension of a semi-simple Lie group by an abelian group (the topological component of the identity of $P \cap R)$. By the Whitehead lemma, the extension almost splits so $P_{0}=(P \cap R)_{0}(S)$ with $S$ and $S \cap(P \cap R)$ finite. The fact that $P_{0}$ is normal implies that $S$ acts trivially on $R /(R \cap P)$ and since $R \cap P$ is central in $G, S$ must centralize $R$. It follows immediately that $S$ is normal in $G$ and that $P_{0}$ is an almost direct product $(U \cap P) \times T_{0} \times S$ and it is quite clear that the algebraic hull of $P$ is at a most finite extension of $P_{0}$ and hence also of $P$. Finally it is clear from all this that $P$ centralizes $U$ and now all statements of the theorem are proved.

\section{REFERENCES}

1. L. Auslander et al., Flows on Homogeneous Spaces, Ann. of Math. Studies No. 53, Princeton Univ. Press, Princeton, N. J., 1963.

2. L. Auslander and L. Green, G-induced flows, Amer. J. Math., 88 (1966), 43-60.

3. L. Auslander, An exposition of the structure of solvmanifolds. Part I; Algebraic theory, Bull. Amer. Math. Soc., 79 (1973), 227-261; Part II, G-induced flows. ibid, 262-285.

4. A. Borel, Linear Algebraic Groups, W. A. Benjamin, New York, 1969.

5. J. Berzin and C. C. Moore, Flows on homogeneous spaces: a new look, to appear.

6. S. G. Dani, Spectrum of an affine transformation, Duke J. Math., 44 (1977), 129-155.

7. I. Gelfand and S. Fomin, Geodesic flows on manifolds of constant negative curvature, Uspekhi Matem. Nauk, 7 (1952), 118-137.

8. R. Howe and C. C. Moore, Asymptotic behavior of unitary representations, J. Functional Analysis, 32 (1979), 72-96.

9. A. A. Kirillov, Unitary representations of nilpotent Lie groups, Uspekhi Matem. Nauk, 17 (1962), 57-110.

10. G. W. Mackey, Induced representations of locally compact groups I, Ann. of Math., 55 (1952), 101-139.

11. Unitary representations of group extensions I, Acta Math., 99 (1958), 265-311.

12. F. I. Mautner, Geodesic flows on symmetric Riemannian spaces, Ann. of Math., 65 (1957), 416-431.

13. C. C. Moore, Ergodicity of flows on homogeneous spaces, Amer. J. Math., 88 (1966), 154-178.

14. - Restrictions of unitary representations to subgroups and ergodic theory, in Group Representations in Mathematics and Physics. Springer Lecture Notes in Physics, No. 6 (1970), 1-17. 
15. A. M. Stepin, Dynamical systems on homogeneous spaces of semi-simple Lie 'groups, Izvestiia Akad. Nauk SSSR, 37 (1973), 1091-1107.

Received March 15, 1979. Supported partially by NSF Grant \#MCS-77-13070 and the Miller Institute for Basic Science.

UNIVERSITY OF CALIFORNIA

BeRKELEY, CA 94720 



\section{PACIFIC JOURNAL OF MATHEMATICS}

\section{EDITORS}

DONALD BABBITT (Managing Editor)

University of Galifornia

Los Angeles, California 90024

HUGo RossI

University of Utah

Salt Lake City, UT 84112

C. C. MOORE AND ANDREW OGG

University of California

Berkeley, CA 94720
J. DUGUNDJI

Department of Mathematics University of Southern California Los Angeles, California 90007

R. FINN AND J. MILGRAM Stanford University Stanford, California 94305

\section{ASSOCIATE EDITORS}

E. F. BECKENBACH

B. H. NEUMANN

F. WOLF

K. YosHIDA

\section{SUPPORTING INSTITUTIONS}

UNIVERSITY OF BRITISH COLUMBIA CALIFORNIA INSTITUTE OF TECHNOLOGY UNIVERSITY OF CALIFORNIA MONTANA STATE UNIVERSITY UNIVERSITY OF NEVADA, RENO NEW MEXICO STATE UNIVERSITY OREGON STATE UNIVERSITY UNIVERSITY OF OREGON
UNIVERSITY OF SOUTHERN CALIFONIA STANFORD UNIVERSITY UNIVERSITY OF HAWAII UNIVERSITY OF TOKYO UNIVERSITY OF UTAH WASHINGTON STATE UNIVERSITY UNIVERSITY OF WASHINGTON 


\section{Pacific Journal of Mathematics}

\section{Vol. 86, No. $1 \quad$ November, 1980}

Gert Einar Torsten Almkvist, Invariants, mostly old ones .............. 1

Hyman Bass, Groups of integral representation type ................ 15

A. Białynicki-Birula, On action of SL(2) on complete algebraic

varieties........................................

Frederick Paul Greenleaf and Martin Allen Moskowitz, Groups of

automorphisms of Lie groups: density properties, bounded orbits, and

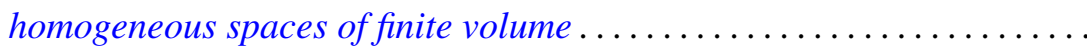

Raymond Taylor Hoobler, A cohomological interpretation of Brauer groups

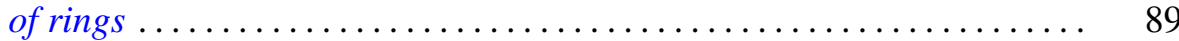

Irving Kaplansky, Superalgebras ........................ 93

Jerrold Lewis Kleinstein and Alex I. Rosenberg, Succinct and

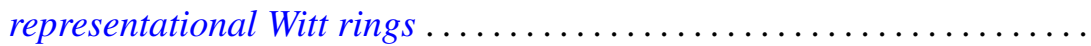

E. R. Kolchin, On universal extensions of differential fields ............ 139

Andy R. Magid, Analytic subgroups of affine algebraic groups. II ....... 145

Calvin Cooper Moore, The Mautner phenomenon for general unitary

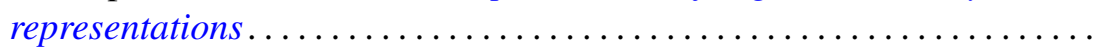

George Daniel Mostow, On a remarkable class of polyhedra in complex hyperbolic space ................................ 171

Brian Lee Peterson, Extensions of pro-affine algebraic groups. II . . . . . . 277

John Henry Reinoehl, Lie algebras and affine algebraic groups......... 287

Maxwell Alexander Rosenlicht, Differential valuations .

John Brendan Sullivan, The second Lie algebra cohomology group and Weyl modules..................................... 321

Moss Eisenberg Sweedler, Right derivations and right differential

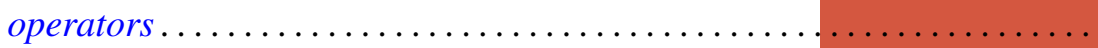

Bostwick Frampton Wyman, Time varying linear discrete-time systems. II. Duality.................................. 